VOL. $5(1971), 387-390$.

\title{
The weak density of the non-invertible elements of a commutative algebra
}

\author{
L. Narici, G. Bachman and E. Beckenstein
}

\begin{abstract}
Let $X$ be a commutative locally convex Hausdorff topological algebra with identity over a non-trivially valued field $F$. Let $M_{c}$ denote the continuous nontrivial homomorphisms of $X$ into $F$ and $M$ the set of all maximal ideals of $X$. If the spectrum of each element $x$ in $X$ is the set of scalars $\left\{f(x) \mid f \in M_{c}\right\}$, it is shown that the singular elements of $X$ are weakly dense in $X$ if and only if $M$ is an infinite set.
\end{abstract}

In this paper we extend the above quoted result, proved for commutative Banach algebras with identity by Graham in [4, p. 179], to a category of topological algebras over arbitrary nontrivially valued fields. Included among these are a number of nonarchimedean locally multiplicatively convex algebras over nonarchimedean valued fields ([3]) such as algebras $F(T)$ of continuous functions from an infinite totally disconnected space $T$ into a complete nonarchimedean valued field $F$ with compact-open topology .

LEMMA 1. If $X$ is a topological algebra over a nontrivially valued field $F$ such that $U M_{c}=U M$, then $M_{c}$ is finite if and only if $M$ is finite.

Proof. Since $M_{c} \subset M$, finiteness of $M$ clearly implies finiteness of $M_{c}$. Conversely we show that if $M_{c}$ is finite, then $M_{c}=M$. To do this let us suppose that $M \in M$ and $M \notin M_{c}$. Letting

Received 7 July 1971. 
$M_{c}=\left\{M_{1}, \ldots, M_{n}\right\}$, then, by hypothesis, $M=\bigcup_{i=1}^{n}\left(M \cap M_{i}\right)$. With no loss of generality we may assume that the above union is irredundant. Thus for each $i, 1 \leq i \leq n$, there must be an element $x_{i} \in \dot{M} \cap M_{i}$ such that $x_{i} \notin M \cap M_{j}$ for $j \neq i$. Consider $y=x_{i}+\prod_{j \neq i} x_{j}$. Since $y \in M$, $y \in M \cap M_{k}$ for some $k, 1 \leq k \leq n$. If $k \neq i$, we are led to the contradictory result: $x_{i}=y-\prod_{j \neq i} x_{j} \in M \cap M_{k}$. If $k=i$ we find that $\prod_{j \neq i} x_{j} \in M \cap M_{i}$. Since $M_{i}$ is a maximal - hence prime - ideal, this too is contradictory. Thus there can be no $M \in M$ such that $M \notin M_{c}$, and the proof is complete.

Clearly $U M=U M_{C}$ in any topological algebra $X$ where the spectrum $\sigma(x)$ of each $x \in X$ satisfies the relationship $\sigma(x)=\left\{f(x) \mid f \in M_{c}\right\}$. We call such algebras Arens algebras. As a result of [1, p. 462] the following algebras are Arens algebras:

(a) any complex commutative complete Hausdorff locally m-convex algebra $([5, p .18])$;

(b) by (a), any complex commutative Banach algebra with identity;

(c) any complete nonarchimedean locally multiplicatively convex algebra over a complete nonarchimedean valued field whose factor algebras are Gelfand algebras ([3], [6, p. 432]).

Taking the radical of $X$ to be $R=n M$, we note that by [8, p. 33], $R=\cap M_{C}$ in any Arens algebra. Before proceeding to the main result, the following lemma is needed.

LEMMA 2. If $X$ is an Arens algebra, then $M$ is infinite if and only if $X / R$ is infinite-dimensional.

Proof. Consider the Gelfand map ([2])

$$
\begin{gathered}
\psi: X \rightarrow E\left(M_{c}\right) \\
x \rightarrow \hat{x}
\end{gathered}
$$


where for any $M \in M_{c}, \hat{x}(M)=x+M$. Since $X$ is an Arens algebra, we observe that $\operatorname{ker} \psi=R$. Thus $X / R$ is isomorphic to $\psi(X)$. If $X / R$ is infinite-dimensional, surely $M_{c}$ is an infinite set. Thus $M$ is infinite. Conversely, by Lemma 1 , if $M$ is infinite, then $M_{C}$ must be infinite. Now $\psi(X)$ is a subalgebra of $F\left(M_{c}\right)$ which separates points of $M_{c}$ so, by elementary considerations, $\psi(X)$ must be infinite-dimensional. Thus $X / R$ is infinite-dimensional.

THEOREM. If $X$ is a locally convex Hausdorff Arens algebra over the reals, the complexes, or any spherically complete valued field, the singular elements of $X$ are weakly dense in $X$ if and only if $M$ is infinite.

Proof. We refer to Graham's proof ([4, p. 180]) making note of the fact that the validity of his proof in this setting depends on the infinite-dimensionality of $X / R$ which has been shown to be true in Lemma 2, the fact that $R=n_{c}$ since $X$ is an Arens algebra, and the availability of the Hahn-Banach Theorem in our setting ( $[7$, p. 78]),

\section{References}

[1] Richard Arens, "A generalization of normed rings", Pacific J. Math. 2 (1952), 455-471.

[2] E. Beckenstein, G. Bachman and L. Narici, "Maximal ideals in topological algebras", J. Analyse Math. 25 (1971), (to appear).

[3] E. Beckenstein, G. Bachman and L. Narici, "Function algebras over valued fields", (to appear).

[4] Colin C. Graham, "The weak density of the non-invertible elements of a commutative Banach algebra", Bull. Austral. Math. Soc. 4 (1971), 179-182.

[5] Ernest A. Michael, "Locally multiplicatively-convex topological algebras", Mem. Amer. Math. Soc. 11 (Amer. Math. Soc., Providence, Rhode Island, 1952). 
[6] Lawrence Narici, "On nonarchimedean Banach algebras", Arch. Math. 19 $(1968), 428-435$.

[7] Lawrence Narici, Edward Beckenstein and George Bachman, Functional analysis and valuation theory (Marcel Dekker, New York, 1971).

[8] W. Żelazko, "Metric generalizations of Banach algebras", Rozprawy Mat. 47 (Polsk. Akad. Nauk, Inst. Mat., Warszawa, 1965).

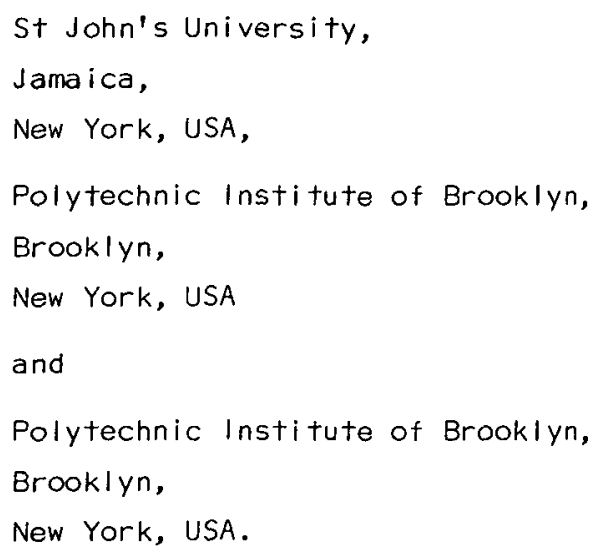

